\title{
Conjunctival involvement in classic (indolent) HIV negative Kaposi's sarcoma
}

\author{
Ilan G Ron, Israel Kremer, Anat Lowenstein, Samario Chaitchik
}

Kaposi's sarcoma can occur in four clinical settings. In the first, the classic or European type, Kaposi's sarcoma is manifested as an indolent cutaneous malignancy primarily involving the lower extremities and feet, and is particularly prevalent among elderly men of Mediterranean or East European Jewish origin. ${ }^{1}$ The second major presentation is the aggressive lymphadenopathic/visceral form found among black Africans (HIV status uncertain). ${ }^{2}$ In the third AIDS related form, the process is both visceral and cutaneous and often aggressive with a poor outlook. ${ }^{3}$ The fourth group is HIV negative, but exogenously immunosuppressed, as in the case of transplant patients. The disseminated, subtype of classic Kaposi's sarcoma is extremely rare. We report herein, to the best of our knowledge, the first case of HIV negative classic type Kaposi's sarcoma involving the conjunctiva, with successful use of cryotherapy.

\section{Case report}

An 83-year-old Jewish male patient of East European origin presented with characteristic crops of violaceous nodular lesions on the skin of his right foot. His general physical examination was unremarkable as was the whole laboratory examination. Blood tests for HIV antibody, and CMV antigen, using enzyme linked immunosorbent assay (ELISA) were negative. Epstein-Barr virus antigen, checked by immunofluorescence, was likewise negative. Testing of serum immunoglobulins, $\mathrm{CD}_{4}$ lymphocyte count, and helper/ suppressor ratio was done, with all results within normal limits. The patient had never received immunosuppressive treatment in the past.

Excisional biopsy of one of his lesions was performed and the histopathological examination revealed the presence of Kaposi's sarcoma stage II of the skin.

Several months later, new lesions appeared on the skin of his right thigh and both scrotums. At

Medical Center

Tel-Aviv, Israel

Department of Oncology

I G Ron

S Chaitchik

Department of

Ophthalmology

A Lowenstein

Department of

Ophthalmology,

Beilinson Medical

Center, Petach Tikva,

Israel

I Kremer

Correspondence to:

Ilan $G$ Ron, $M D$, Department of Oncology, Tel-Aviv

Sourasky Medical Center

6 Wrasky Medical Center, 64239 , Israel.

Accepted for publication

26 January 1994

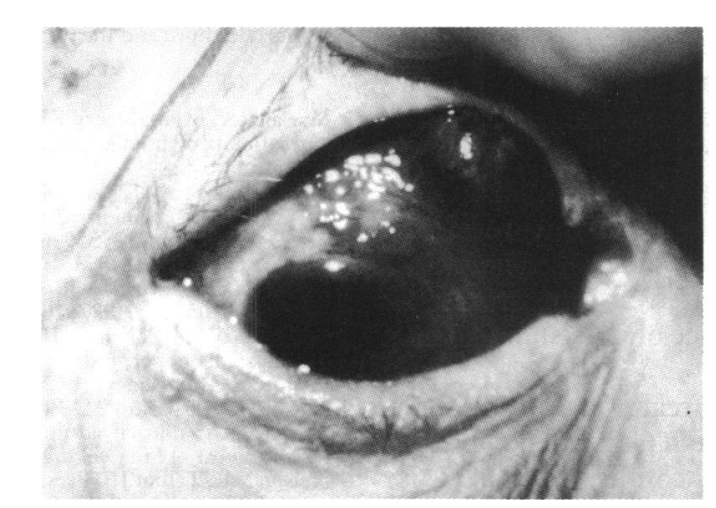

Figure 1. Stage III classic type Kaposi's sarcoma in right conjunctiva.

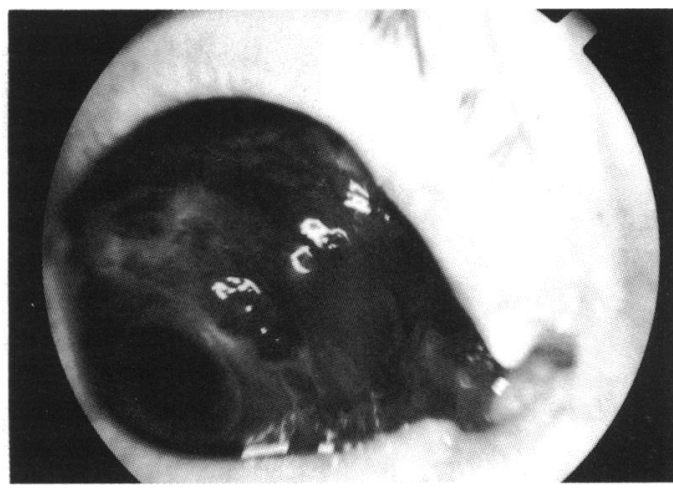

Figure 2 Significant regression of the Kaposi's lesions at the end of cryotherapy treatments (photograph taken 10 days following last treatment).

the same time, several dark red coloured $3 \times 3$ $\mathrm{mm}$ nodular elevated lesions were found in the right bulbar and palpebral conjunctiva (Fig 1). Subsequently, the patient complained of marked discomfort and tearing. The ophthalmic examination of the right eye revealed a clear cornea, pseudophakia, and normal fundus. Examination of the left eye was unremarkable. The patient was treated by cryoapplication with $-80^{\circ} \mathrm{C}$ probe. Each lesion was treated with three applications of 20 seconds each, once a week for 4 weeks until significant regression was achieved (Fig 2).

At the end of a 2 year follow up, no evidence of systemic involvement was found and the conjunctival lesions did not recur following the cryotherapy (Fig 3).

\section{Comment}

Kaposi's sarcoma is a multicentric vascular neoplasm affecting the skin, mucous membranes, internal organs, and lymph nodes. It is usually associated with AIDS, involving $15 \%$ to $24 \%$ of AIDS patients. ${ }^{4}$

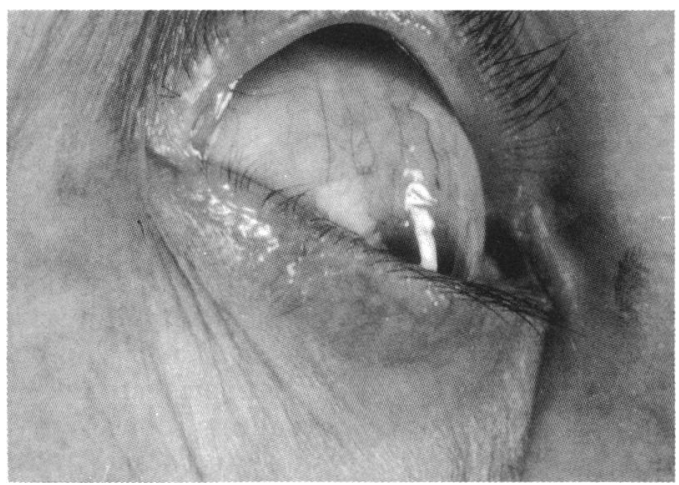

Figure 3 Treated right eye showing no recurrences of Kaposi's lesions in the conjunctiva, at 2 year follow up. 
AIDS related Kaposi's sarcoma follows a much more fulminant course and is less responsive to therapy than the more rare indolent HIV negative Kaposi's sarcoma, which affects elderly males of Mediterranean or East European Jewish origin or the type which appears in young adult black African males. ${ }^{1}$ The therapeutic options for adnexal ocular Kaposi's sarcoma are: (a) observation of asymptomatic lesions; (b) surgical excision; (c) cryotherapy; (d) irradiation. ${ }^{56}$

Dugel et $a l^{5}$ classified conjunctival Kaposi lesions into three clinical stages: stage I and II are patchy and flat (less than $3 \mathrm{~mm}$ in height); stage III lesions are more nodular and more than $3 \mathrm{~mm}$ in height. ${ }^{5}$ They found a better rate of success for surgical excision and/or cryotherapy for stage I and II tumours.

Because our patient's conjunctiva was affected by several lesions, necessitating excision of a large area of the conjunctiva, and because of the benign course of this type of Kaposi's sarcoma, we chose the cryotherapy option of treatment. It is also obvious that the risk/benefit ratio of this method is much lower than that of radiotherapy of the adnexa oculi.

This patient is unique as his disease course was benign, his immunological profile was normal, he had never received immunosuppressive therapy, and his blood tests were negative for HIV antigens.

1 Friedman-Krien AE, Saltzman BR. Clinical manifestations of classical endemic African and epidemic AIDS-related Kaposi's sarcoma. F Am Acad Dermatol 1990; 22: 1237-50.

2 DiGiovanna JJ, Safai B. Kaposi's sarcoma: retrospective study of 90 cases with particular emphasis on the familial occurrence, ethnic background and prevalence of other diseases. Am F Med 1981; 71: 779-83.

3 Ziegler JL, Tempelton AC, Vogel CL. Kaposi's sarcoma: a comparison of classical, endemic and epidemic forms. Semin Oncol 1984; 11: 47-52.

4 Haverkes HW, Friedman-Krien AE, Drotman DP, Morgan WM. The changing incidence of Kaposi's sarcoma amongs patients with AIDS. 7 Am Acad Dermatol 1990; 22: 1250-3.

5 Dugel PU, Gill PS, Frangieh GT, Rao NA. Treatment of ocular adnexal Kaposi's sarcoma in acquired immune deficiency syndrome. Ophthalmology 1992;99: 1127-32.

6 Gabrial L, Quivey JM, Dunn JP, Char DH. Radiation therapy of acquired immunodeficiency syndrome-related Kaposi's sarcoma of the eyelids and conjunctiva. Arch Ophthalmo 1992; 110: 1423-6.

\title{
Acinetobacter exposure keratitis
}

\author{
A Marcovich, S Levartovsky
}

Department of

Ophthalmology, Kaplan

Hospital, Rehovot and the Hebrew University, Hadassah Medical

School, Jerusalem, Israel

A Marcovich

S Levartovsky

Correspondence to:

Arie Marcovich, MD

Department of

Ophthalmology, Kaplan

Hospital, Rehovot 76110,

Israel.

Accepted for publication

26 January 1994
Acinetobacter is a pleomorphic Gram negative bacterium. Different names have been used to describe the organism. Recently its taxonomy has changed considerably. Acinetobacter strains are now referred to as the Acinetobacter calcoaceticusA baumannii complex. 'Acinetobacter keratitis is rare. Acinetobacter keratitis has been reported in association with soft contact lens wear, ${ }^{23}$ penetrating keratoplasty, ${ }^{4}$ and in a patient with chronic lymphatic leukaemia. ${ }^{5}$ Corneal perforation caused by Acinetobacter has been described. ${ }^{6}$ We report a case of corneal ulcer caused by Acinetobacter calcoaceticus- $A$ baumannii complex in a gravely ill patient who also suffered from exposure keratitis.

\section{Case report}

A 74-year-old man with chronic lung disease, diabetes mellitus, and right hemiparesis secondary to an old cerebrovascular accident was admitted to Kaplan Hospital with pneumonia, urinary tract infection, cachexia, and deep vein thrombosis. He improved after 12 days of intravenous cefuroxime and was discharged in good health. $\mathrm{He}$ was readmitted 1 week later with fever, cough, and general deterioration. Another course of treatment with intravenous cefuroxime improved the patient's status but 4 days later the right eye became inflamed. On examination the patient exhibited minimal blinking movements which led to exposure keratitis. A corneal infiltrate in the lower mid-periphery as well as an abundant conjunctival discharge were present. There was no anterior chamber reaction. The corneal ulcer was scraped and cultured. No organisms were detected in the Gram or Giemsa stained smears. The patient was treated with gentamicin $(15 \mathrm{mg} / \mathrm{ml})$ and cephazolin $(50 \mathrm{mg} / \mathrm{ml})$ eyedrops hourly and tarsorrhaphy was performed. There was marked improvement during the following days. A glucose oxidising member of the Acinetobacter calcoaceticus $-A$ baumannii complex grew out of the culture which was sensitive to imipenem, gentamicin, tobramycin, polymyxin B, doxycycline, minocycline, tetracycline, cotrimoxazole, and sulfa. The isolate was resistant to ampicillin, mezlocillin, piperacillin, aztreonam, bacitracin, the cephalosporins, amikacin, neomycin, chloramphenicol, clindamycin, ciprofloxacin, and ofloxacin. After 5 days of treatment the corneal ulcer cleared. Acinetobacter, demonstrating the same sensitivity profile as in the corneal ulcer, was also isolated in the urine. Two days later the patient became febrile; a chest $x$ ray revealed bilateral lung infiltrates. Despite treatment with intravenous chloramphenicol, the patient's status continued to deteriorate and he died. Permission for autopsy was not granted. 